\title{
Comparison of Spatial Regression Models with Road Traffic Accidents Data
}

\author{
Ghanim Al-Hasani, Md Asaduzzaman, Abdel-Hamid Soliman \\ School of Creative Arts and Engineering \\ Staffordshire University \\ College Road, Stoke-on-Trent, UK \\ ghanimalikhalfansalim.al-hasani@research.staffs.ac.uk; Md.Asaduzzaman@staffs.ac.uk; a.soliman@staffs.ac.uk
}

\begin{abstract}
Road traffic accidents (RTA) cause severe problems for the societies in developed and developing countries and result in loss of lives and highly burden cost. Investigators have applied a wide variety of methodological techniques over the years in order to gain more understanding in this discipline. Spatial models, an elegant technique to model spatial data, has been applied to capture localisation effects and influencing factors on RTA. This study aims to compare the spatial lag model (SLM) and the spatial error model (SEM) with the Ordinary least square (OLS) model for the road traffic accident data in the Sultanate of Oman. To compare the performance and accuracy of the OLS, SLM and SEM models, the log-likelihood, Akaike information criterion (AIC), and Bayesian information criteria (BIC) have been used. The SLM model outperforms SEM and OLS models for the data and one of the most significant findings to emerge from this study as the SLM model provides the best values of log-likelihood, AIC and BIC comparing to the values from OLS and SEM models.
\end{abstract}

Keywords: Road traffic accidents (RTA); Spatial modelling; Spatial regression; Comparison of models.

\section{Introduction}

With a view to decrease the road burden, the origin and nature of road traffic accidents (RTA) have been theoretically and empirically investigated by many researchers [1]. Although statistical modelling for RTA has improved significantly in the past years, there are still many gaps to be filled between practical analysis and the frontier analysis [2]. Generalised linear modelling (GLM) is one of the most popular techniques used for the RTA analysis. However, the GLM has a strong drawback as the model cannot capture spatial correlations existing in the RTA data [3]. Many methodological advances on spatial analysis have been developed to treat the subtle issues in traffic data, for instance, the impact of unobserved factors in spatial correlations, accident frequencies, unobserved heterogeneity, endogeneity, etc [2].

Spatial models are popular methodological techniques which have gained much attention for empirical research recently including road traffic accident analysis. The spatial methods have been applied to capture spatial effects and influencing factors for many RTA research. In the spatial investigation of RTA, the impact of population, density, number of vehicles, etc. on the number of accidents have been performed taking regions as the spatial units. However, identification or the justification of the factors are critical for spatial models. [5] indicated that improved prediction can be achieved by examining and control of the multiple spatial factors in road safety studies. Although there are many studies in accident research and many models have been suggested for each type of dataset, it is painful and crucial to choose an appropriate model due to insufficient guidelines regarding spatial model selection. Equally, [6] argues that the estimation of spatial model parameters could be insignificant based on spatial model residuals according to spatial units levels in that model. However, the parameter estimation, hypothesis testing, model diagnostics, etc. are equally crucial for model fitting with spatial data. Moreover, model misspecification with the potential spatial correlation for the spatial models increases model residuals [7]. As such, popular spatial models applied in RTA are a spatial lag model (SLM) and spatial error models (SEM).

Investigators carried out a number of studies on road traffic accidents (RTA); however, they investigated different aspects for different countries. In Oman, roads are the only possible option for transport both in terms of human occupants or goods to be transported instead of other transports like railways in many other countries. As a result, RTA has been a prominent public health problem in the country [9]. The spatial units of this analysis are the 11 governorates in the Sultanate of Oman. Although some researchers have been carried out studies on the road safety of Oman, to the best of our knowledge, no study has been found that applies SLM or SEM with RTA data in Oman. Therefore, the purpose of this study is to compare the most popular spatial models for the road traffic accident and find the one that suits the most for the RTA data in Oman. However, [5] found that SEM model performs better than SLM and OLS for an accidents dataset for Seoul, Korea. In a similar study, [6] applied both models (SLM and SEM) to analyse data for 633 census wards in London metropolitan regions. However, due to the different choice of high-level spatial units such as regions or Governorates, the model performance varies significantly.

In this paper, we compare the spatial lag model (SLM) and the spatial error model (SEM) with the Ordinary least square (OLS) model for the RTA data in Oman to compare the performance and find the most suitable one. To evaluate and diagnose the accuracy of three models (OLS, SLM and SEM) we checked the model residuals, and use three diagnostic tools: log-likelihood, Akaike information criterion (AIC) and Bayesian Information Criteria (BIC). The rest of the paper is organised as the methodology in Section 2, results and discussion are given in Section 3 and some concluding remarks in Section 4. 


\section{Methdology \\ 2.1. Models}

Three models- Ordinary least square model (OLS), spatial lag model (SLM) and spatial error model (SEM) are applied in this study with the spatial data to compare their performance and accuracy. The ordinary least square model can be written as

$$
Y_{i}=\beta_{i} X_{i}+\xi,
$$

where $Y_{i}$ is the number of RTAs as the dependent variable for $i=1,2, \ldots, n, \beta_{i}$ is a vector of parameters, $X_{i}$ is a matrix of independent variables and, $\xi$ is a vector of unobserved error terms that assumed to be distributed normally $N\left(0, \sigma^{2}\right)$. The estimates of parameters $\beta$, in matrix notation, [10] can be given as

$$
\hat{\beta}_{i}=\left(X^{t} X\right)^{-1} X^{t} Y,
$$

where $X^{t}$ is the transpose of data matrix, $X$, and $Y$ is the observed vector of the dependent variable.

In this study, we evaluate two spatial models SLM and SEM, where the neighbouring (spatial) effect for a region $i$ is considered to be affected by the other neighbouring regions $j, j=1,2, \ldots, n[11]$. Let us define the spatial dependence as

$$
Y_{i}=f\left(Y_{j}\right), \quad i, j=1,2, \ldots, n ; i \neq j,
$$

where $Y_{i}$ is the natural logarithm of the number of RTAs in $i$ spatial units. However, this study applied two spatial models (SLM and SEM) compared with the OLS model as given in Eq (1).

The spatial lag model (SLM) models can be written as

$$
Y_{i}=\rho W_{y}+\beta_{i} X_{i}+\xi,
$$

where $Y_{i}$ is the vector of natural logarithm of RTAs for $i$ th spatial unit (region), $\rho$ is the spatial lag coefficient, $W_{y}$ is the spatial weight matrix, $X_{i}$ is the matrix of independent variables, $\beta_{i}$ is the vector of parameters and $\xi$ is the unobserved error terms vector.

The spatial error model (SEM) can be written as

$$
Y_{i}=\beta_{i} X_{i}+\mu, \text { with } \mu=\lambda W_{\mu}+\xi,
$$

where $\mu$ is the function of unobserved error terms, $\lambda$ is a spatial error coefficient and $W_{\mu}$ is the spatial error weight matrix. The spatial weights are distinct elements in any cross-sectional analysis of spatial dependence. They are necessary components in the setting up of spatial autocorrelation statistics and provide calibration between units. For both SLM and SEM, the spatial weight matrix $W$ can be defined as

$$
W=\left[\begin{array}{cccc}
W_{11} & W_{12} & \ldots & W_{1 n} \\
W_{21} & W_{22} & \ldots & W_{2 n} \\
\vdots & \vdots & \ddots & \vdots \\
W_{n 1} & W_{n 2} & \ldots & W_{n n}
\end{array}\right] .
$$

\subsection{Data}

Data for this study have been gathered from multiple sources. RTA data have been collected from the published reports by Royal Omani Police [12]. Other secondary data on different factors such as population, area, density, etc. from the publication of the National Centre for Statistics \& Information (NCSI) in Oman [13, 14, 15, 16]. Unemployment statistics were provided by the Public Authority of Manpower Register in Oman.

In this study, the number of road traffic accidents in Oman in 2017 is considered as the dependent variable and the independent variables are density, population, number of vehicles, number of job seekers (unemployment), number of accidents caused by the speed in 2017 and number of accidents occurring during seasonal months (May, June, July 2017). There are eleven Governorates in Oman which represent the spatial units in this study.

\section{Results and Discussion}

In this study, three models have been applied which are ordinary least square (OLS), spatial lag model (SLM) and spatial error model (SEM) to the RTA data of Oman for the year 2017. In the regression models, the number of road traffic accidents in different Governorates (regions) in Oman is considered as the dependent variable. The independent variables in this study are density, population, number of vehicles, number of job seekers (unemployment), number of accidents caused by speed in 2017 and number of accidents occurring during seasonal months (May, June, July 2017) in the Sultanate of Oman. There are eleven Governorates in Oman are representing the spatial units in this study. The results are showed in Table 1 that are obtained from the fitting of the three models- OLS, SLM and SEM.

The results showing in the Table 1 gives the comparison of the estimated parameters and their significance levels $(*: P<0.05$, $* *: P<0.001, * * *: P<0.0001$ ). The effects of two variables (number of speed accidents and seasonal accidents) obtained are similar in all three models while there is a clear difference in density, population and number of vehicles. Spatial error model gives very close coefficient to ordinary least square to the number of job seekers variable.

For checking the adequacy of models, the residual plots can be used. The residuals of the three models were also evaluated as displayed in Fig 1. This suggests that the SLM for RTAs data has higher accuracy than others. To select the most suitable model, Akaike information criteria (AIC) and Bayesian information criteria (DIC) are the two major criteria widely used including spatial data analysis [11]. 
Table 1: Results of parameter estimates in different spatial models for RTA data.

\begin{tabular}{l|lll}
\hline Dependent variable & OLS & SLM & SEM \\
\hline Spatial lag coefficient, $\rho$ & - & 0.061498 & - \\
Spatial error coefficient, $\lambda$ & - & - & -1.5208 \\
Constant & $-1.96 \mathrm{E}+01 * *$ & $-3.76 \mathrm{E}+01 * * *$ & $-2.06 \mathrm{E}+01 * * *$ \\
Density & $4.90 \mathrm{E}-01 *$ & $2.39 \mathrm{E}-01 * * *$ & $5.11 \mathrm{E}-01 * * *$ \\
Population & $2.85 \mathrm{E}-05$ & $-3.76 \mathrm{E}-05^{* * *}$ & $4.40 \mathrm{E}-05 * * *$ \\
Number of vehicles & $-3.99 \mathrm{E}-04 * *$ & $-2.20 \mathrm{E}-04 * * *$ & $-4.04 \mathrm{E}-04 * * *$ \\
Number of job seekers & $-1.10 \mathrm{E}-03$ & $-1.07 \mathrm{E}-04$ & $-1.20 \mathrm{E}-03 *$ \\
Number of speed accidents & $6.64 \mathrm{E}-01^{* *} *$ & $6.00 \mathrm{E}-01 * * *$ & $6.53 \mathrm{E}-01 * * *$ \\
Number od seasonal accidents & $2.56 \mathrm{E}+00^{* * *} *$ & $2.76 \mathrm{E}+00^{* * *}$ & $2.53 \mathrm{E}+00^{* * *}$ \\
\hline \multicolumn{2}{c}{ Level of signficance: $*: P<0.05, * *: P<0.001, * * *: P<0.0001$}
\end{tabular}
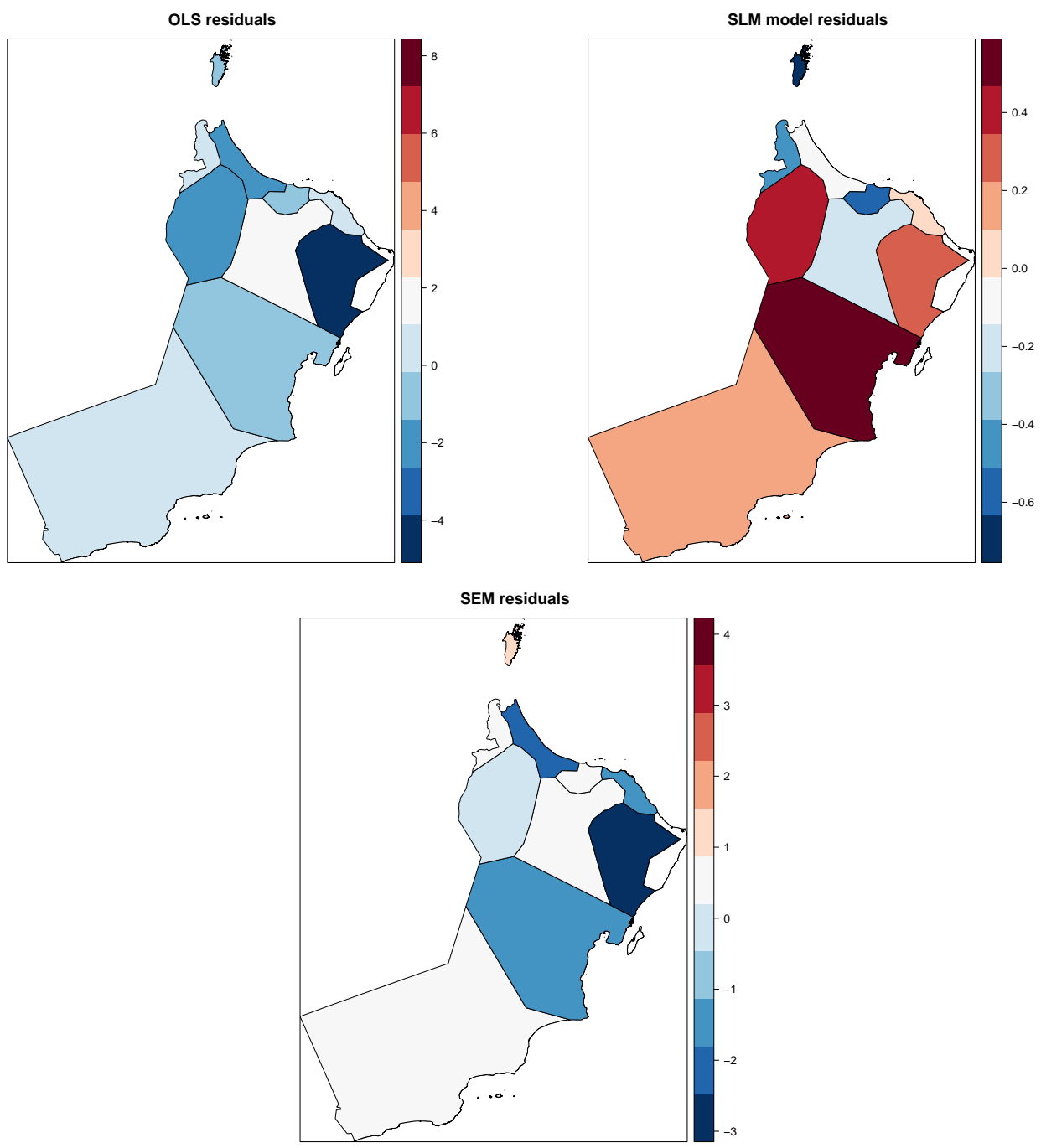

Fig. 1: Models residuals through Oman's Governorates.

As showing in Table 2, the values of the log-likelihood, AIC and BIC of OLS, SLM and SEM models were compared in order to determine the best model. The most interesting finding is that the SLM outperforms the SEM due to best values of log-likelihood $=-6.34, \mathrm{AIC}=30.69$ and $\mathrm{BIC}=34.27$ while the corresponding values for SEM are $-25.78,69.55$ and 73.13 , respectively. Therefore, the SLM model found to be the best to identify associated factors for the road traffic accidents in Oman. 
Table 2: Evaluation of different spatial models for RTA data.

\begin{tabular}{c|ccc}
\hline Diagnostic Criteria & OLS & SLM & SEM \\
\hline log-likelihood & -28.18 & -6.34 & -25.78 \\
AIC & 72.37 & 30.69 & 69.55 \\
BIC & 75.54 & 34.27 & 73.13 \\
& & & \\
\hline
\end{tabular}

\section{Conclusion}

The main goal of the current study is to compare the spatial lag model (SLM) and the spatial error model (SEM) with road traffic accidents (RTA) data. The study evaluated and diagnosed three models, which are OLS, SLM and, SEM. The study showed that the similar impact of three models OLS, SLM and SEM in two variables; the number of speed accidents and seasonal accidents. In contrast, it was shown a clear difference on the effect of the variables such as density, population and number of vehicles in all three models. One of the significant findings to emerge from this study is that the SLM outperformed the SEM due to the best values in log-likelihood, AIC and, BIC. However, study [5] found the SEM has higher accurancy than SLM model for RTA with different spatial dataset while considered smaller spatial units. Whilst this study did not conform to the finding of the study by [5], this study yet offers some model selection techniques for spatial analysis.

\section{Acknowledgments}

The authors would like to thank the Academic Skill Tutors at the Staffordshire University Library for their support. We also thank the Royal Omani Police (ROP), Authority of Manpower Register and the National Centre for Statistics \& Information NCSI in the Sultanate of Oman for providing support to gather data.

\section{References}

[1] H. Al Reesi, J. Freeman, J. Davey, S. Al Adawi, and A. Al Maniri, "Measuring risky driving behaviours among young drivers: Development of a scale for the Oman setting," Transportation Research Part F: Traffic Psychology and Behaviour, vol. 55, pp. $78-89,2018$.

[2] F. Mannering and C. Bhat, "Analytic methods in accident research: methodological frontier and future directions," Analytic Methods in Accident Research, vol. 1, pp. 1-22, 2014.

[3] Z. Li, W. Wang, P. Liu, J. M. Bigham, and D. R. Ragland, "Using geographically weighted poisson regression for county-level crash modeling in california," Safety science, vol. 58, pp. 89-97, 2013.

[4] S. Barua, K. El-Basyouny, and M. T. Islam, "Effects of spatial correlation in random parameters collision count-data models," Analytic Methods in Accident Research, vol. 5, pp. 28-42, 2015.

[5] K.-A. Rhee, J.-K. Kim, Y.-I. Lee, and G. F. Ulfarsson, "Spatial regression analysis of traffic crashes in seoul," Accident Analysis \& Prevention, vol. 91, pp. 190-199, 2016.

[6] M. A. Quddus, "Modelling area-wide count outcomes with spatial correlation and heterogeneity: An analysis of london crash data," Accident Analysis \& Prevention, vol. 40, no. 4, pp. 1486-1497, 2008.

[7] J. Aguero-Valverde and P. Jovanis, "Analysis of road crash frequency with spatial models," Transportation Research Record: Journal of the Transportation Research Board, no. 2061, pp. 55-63, 2008.

[8] B. Lu, P. Harris, M. Charlton, and C. Brunsdon, "The gwmodel r package: further topics for exploring spatial heterogeneity using geographically weighted models," Geo-spatial Information Science, vol. 17, no. 2, pp. 85-101, 2014.

[9] A. A. N. Al-Maniri, H. Al-Reesi, I. Al-Zakwani, and M. Nasrullah, "Road traffic fatalities in Oman from 1995 to 2009: evidence from police reports," International Journal of Preventive Medicine, vol. 4, no. 6, p. 656, 2013.

[10] C. Brunsdon, A. S. Fotheringham, and M. E. Charlton, "Geographically weighted regression: a method for exploring spatial nonstationarity," Geographical analysis, vol. 28, no. 4, pp. 281-298, 1996.

[11] L. Anselin, "Model validation in spatial econometrics: a review and evaluation of alternative approaches," International Regional Science Review, vol. 11, no. 3, pp. 279-316, 1988.

[12] R. O. Police, Facts and Figures. Director General of Traffic, 2017.

[13] NCSI, Monthly Statistical Bulletin April 2017. National Centre for Statistics \& Information, Sultanate of Oman, 2017.

[14] — Monthly Statistical Bulletin July 2017. National Centre for Statistics \& Information, Sultanate of Oman, 2017.

[15] —-, Monthly Statistical Bulletin September 2017. National Centre for Statistics \& Information, Sultanate of Oman, 2017.

[16] — Monthly Statistical Bulletin January 2018. National Centre for Statistics \& Information, Sultanate of Oman, 2018. 